\title{
Indicators of quality of care for patients with acute myocardial infarction
}

\author{
Jack V. Tu MD PhD, Laila Khalid MD, Linda R. Donovan BScN MBA, Dennis T. Ko MD MSc, \\ for the Canadian Cardiovascular Outcomes Research Team / Canadian Cardiovascular Society \\ Acute Myocardial Infarction Quality Indicator Panel
}

$\infty \infty \quad$ See related research, pages 895 and 901, and related commentary, page 875

\section{ABSTRACT}

Background: There is a wide practice gap between optimal and actual care for patients with acute myocardial infarction in hospitals around the world. We undertook this initiative to develop an updated set of evidence-based indicators to measure and improve the quality of care for this patient population.

Methods: A 12-member expert panel was convened in 2007 to develop an updated set of quality indicators for acute myocardial infarction. The panel identified a list of potential indicators after reviewing the scientific literature, clinical practice guidelines and other published quality indicators. To develop the new list of indicators, the panel rated each potential indicator on 4 dimensions (reliability, validity, feasibility and usefulness in improving patient outcomes) and discussed the topranked quality indicators at a consensus meeting.

Results: Consensus was reached on 38 quality indicators: 17 that would be measurable using chart-abstracted data and 21 that would be measurable using administrative data. Of the 17 chart-review indicators, 13 address pharmacologic and nonpharmacologic care delivered to patients in hospital. In-hospital mortality was recommended as a key outcome indicator. Three system indicators were recommended to measure the collaborative responsiveness of the health care system from the call for help to intervention. It was recommended that hospitals strive for a minimum target benchmark of $90 \%$ or greater on process-of-care indicators.

Interpretation: Implementation of strategies by clinicians and hospitals to meet target benchmarks on these quality indicators could save the lives of many individuals with acute myocardial infarction.

Une version française de ce résumé est disponible à l'adresse www.cmaj.ca/cgi/content/full/179/9/909/DC1

CMAJ 2008;179(9):909-15

$\mathrm{T}$ here is a large practice gap between optimal and actual patterns of care for patients with acute myocardial infarction in hospitals around the world. ${ }^{1}$ Acute myocardial infarction is a highly treatable condition for which many advances in treatment have occurred over the past several decades. However, the uptake of many of these advances and their incorporation into routine clinical practice has often lagged behind their development and publication in clinical journals by many years. ${ }^{2-4}$ To reduce this gap and improve quality of care, many jurisdictions are using indicators of the quality of care for patients with acute myocardial infarction. These quality indicators are intended to measure adherence to selected key clinical practice guidelines in routine clinical care and serve as a foundation for efforts to improve quality. ${ }^{5}$ They define the minimum standard of care that might be expected for all "ideal" patients who meet certain criteria and have no contraindications for a given health care intervention.

National organizations in Canada, the United States, the United Kingdom and other OECD (the Organisation for Economic Co-operation and Development) countries have all developed indicators to measure and improve the quality of care for patients with acute myocardial infarction both within and across countries, regions and hospitals. ${ }^{6-9}$ In 2003, the Canadian Cardiovascular Outcomes Research Team (a Canadian Institutes of Health Research Interdisciplinary Health Research Team) worked in association with the Canadian Cardiovascular Society to develop and publish the first set of quality indicators for myocardial infarction in Canada. ${ }^{6}$ The Canadian Cardiovascular Outcomes Research Team comprises more than 30 leading outcome researchers who work together on projects to measure and improve cardiac care. From the outset, we recognized that the indicators would need to be modified over time to reflect changes in practice guidelines and clinical evidence. In 2007, a Canadian expert panel was convened by the Canadian Cardiovascular Outcomes Research Team to develop and recommend a set of quality indicators that took into account the best indicators developed elsewhere and that also included some unique indicators that were felt to be of particular relevance and use to Canadian clinicians and hospitals.

\section{Methods}

\section{Expert panel}

A 12-member multidisciplinary expert panel was assembled in 2007 from across Canada to review and update the 2003 indi-

From the Institute for Clinical Evaluative Sciences (Tu, Khalid, Donovan, Ko), Toronto, Ont.; the Division of Cardiology, Schulich Heart Centre, Sunnybrook Health Sciences Centre (Tu, Ko), Toronto, Ont.; and the Department of Medicine, Sunnybrook Health Sciences Centre, University of Toronto ( $\mathrm{Tu}$, Ko), Toronto, Ont. Members of the panel appear at the end of the article. 
cators of quality of care for patients with acute myocardial infarction. ${ }^{6}$ The indicators were developed by means of a twostaged modified Delphi process. ${ }^{10}$ The panel was led by 2 experienced clinician-scientist cochairs (J.V.T. and D.T.K.) and comprised 10 panelists: 3 cardiologists, 2 emergency physicians, 2 registered nurses, a family practitioner, a clinical pharmacist and a clinical epidemiologist (a list of the panel members appears at the end of the article). Panel members were invited by the 2 cochairs based on nominations received from the Canadian Cardiovascular Outcomes Research Team and the Canadian Cardiovascular Society. An attempt was made to create an expert panel that was geographically representative of Canada, was multidisciplinary and included not only experts in cardiology but also leaders in quality improvement with experience in measuring and using indicators of quality of care for patients with acute myocardial infarction.

\section{Review of existing indicators}

In preparation for the update and revision process, a literature search was conducted of the MEDLINE database (1966 onward) to identify articles on indicators of quality of care for acute myocardial infarction. The following search terms were used: "performance indicators," "quality indicators," "performance measures," "quality measures," "report card," "registry," "acute myocardial infarction," "acute coronary syndrome," "cardiology," "healthcare," "benchmarks" and "standards." In addition, bibliographies of relevant articles and websites of specific organizations (e.g., the Joint Commission on Accreditation of Healthcare Organizations and the Centers for Medicare and Medicaid Services) and those involved in quality improvement in different jurisdictions such as the United States, the United Kingdom, Sweden and other countries were identified. Previously published quality indicators, including the 2006 American College of Cardiology / American Heart Association clinical performance measures for myocardial infarction, ${ }^{7}$ were also reviewed. The review of existing indicators, restricted to those published in English, was conducted by one of the coauthors (L.K.) and reviewed by the panel cochairs (J.V.T. and D.T.K.). The results from this literature review appear in Appendix 1 (available online at www.cmaj.ca/cgi/content/full/179/9/909/DC2).

Panel members were encouraged to develop indicators that reflected class I recommendations in the American College of Cardiology / American Heart Association guidelines. Recommendations from the 2004 American College of Cardiology / American Heart Association guidelines for the management of patients with ST-segment elevation myocardial infarction, ${ }^{11}$ and the 2007 American College of Cardiology / American Heart Association guidelines for the management of patients with unstable angina and non-ST-segment elevation myocardial infarction ${ }^{12}$ were used as reference material during the development of the indicators. The 2007 focused update of the 2004 American College of Cardiology / American Heart Association guidelines for the management of patients with STsegment elevation myocardial infarction ${ }^{13}$ was published after the expert panel meeting, but the recommendations were reviewed during the process of finalizing the indicators.

After reviewing the scientific literature, practice guidelines and existing indicators, the panel formulated a new list of potential indicators. Useful indicators are described as "meaningful, valid and reliable, feasible and accountable for patient variability" and capable of being modified by improvements in the health care system. ${ }^{14}$ In addition, the indicators should be measurable using either data available from chart review or linked administrative data sets. ${ }^{6}$ The panel classified indicators by data source (i.e., chart review or administrative data) because administrative data may be quite different from data determined through chart review. Indicators based on data from chart review are more likely to be measured by clinicians working in hospitals, whereas indicators based on administrative data are more likely to be used by government agencies and researchers with access to these data. Although structural indicators were included in the 2003 set of quality indicators, ${ }^{6}$ they were not widely used by Canadian hospitals after publication. As a result, it was decided that the update process would focus on 2 types of indicators: process-of-care indicators and outcome indicators.

The panel divided the process-of-care indicators further into 2 subgroups, according to the relevant stage of care - in hospital and out of hospital. An additional third group was created for "system" indicators that measure aspects of care reflective of the collaborative performance of the health care system from the prehospital to the in-hospital phase; the panel recognized that these indicators are not totally under the control of hospitals. The definitions of the process-of-care indicators included the concept of "eligible" patients (those with a diagnosis of acute myocardial infarction who meet minimal eligibility criteria for an intervention) and "ideal" patients (eligible patients with no contraindications to the intervention of interest).

\section{Preliminary rating of indicators}

The 10 panelists completed the first round of rating the potential indicators independently in September 2007. Each panelist was provided with background material, including the results of the literature review, the original list of quality indicators $^{6}$ and a list of 56 potential indicators (45 process-ofcare indicators, 9 outcome indicators and 2 system indicators [see Appendix 2, available at www.cmaj.ca/cgi/content/full /179/9/909/DC2]) and their associated operational definitions. Panelists were asked to rate each potential indicator according to 4 dimensions recommended by the American College of Cardiology / American Heart Association performance measures task force: usefulness in improving patient outcomes; feasibility of data collection; reliability; and validity. ${ }^{5}$ In addition, each panel member was asked to rate each indicator on a 3-point scale: "do not include," "could include" and "must include." In order for an indicator to be accepted into the second round of rating, a minimum of 7 of the 10 panelists needed to rate it as "must include." The rating forms included a comments section to encourage panelists to provide suggestions for changes or additional indicators. A total of 17 additional indicators suggested by the panelists were assessed by the panel cochairs. Two were found to have sufficient scientific evidence to warrant their inclusion as new indicators in the second round of rating.

Of the 56 potential indicators provided to the panelists for 
the first round of rating, 21 indicators based on administrative data received unanimous ratings of "must include." These 21 indicators were marked for inclusion in the final set of updated indicators and were excluded from the second round of rating.

\section{Secondary rating of indicators}

Panelists participated in a second round of indicator ratings at an expert panel meeting held in Toronto in October 2007 moderated by the cochairs. Thirty-two indicators were presented for the second round of rating. For each indicator, a summary of first-round ratings, suggested changes, comparative indicator definitions used in other jurisdictions and current evidence from practice guidelines were presented and reviewed. This step was followed by discussion of the indicator definition, inclusion and exclusion criteria, feasibility and the indicator's relevance to the Canadian health care system. In many cases, the panel adopted indicator definitions similar to those used in other jurisdictions such as the United States or the United Kingdom where it felt the definitions were suitable for use within a Canadian context. The panelists also discussed target benchmarks. At the end of the discussion process, the panelists were requested to rate each indicator and identify a benchmark.

\section{Final review}

Following the panel meeting, the 32 indicators reviewed were documented as per panel discussions and the overall ratings compiled. The prespecified criterion for inclusion in the final set of indicators was a "must include" rating by at least 7 of the 10 panelists after the expert panel meeting. A summary document of the finalized set of indicators, including any further evidence published in the interim period, was circulated to the panel members. A follow-up teleconference was held to address any outstanding issues.

\section{Results}

\section{Process-of-care indicators - chart-abstracted data}

The expert panel reached a consensus after 2 rounds of ratings on 13 in-hospital process-of-care indicators (6 pharmacologic and 7 nonpharmacologic), 1 outcome indicator and 3 system indicators (Box 1) (Appendix 3, available online at www.cmaj.ca /cgi/content/full/179/9/909/DC2). These indicators are based on chart-abstracted data.

Of the 6 pharmacologic indicators, 2 focus on treatment provided on arrival at hospital (acetylsalicylic acid [ASA] and fibrinolytic therapy) and 4 focus on treatment at discharge (ASA, $\beta$-blocker, angiotensin-converting-enzyme [ACE] inhibitor or angiotensin-receptor blocker, and statin). The 6 pharmacologic indicators are similar to ones used in the United States and are supported by class I recommendations from the American College of Cardiology / American Heart Association 2004 and 2007 guidelines. ${ }^{11-13}$ However, the first indicator — "ASA prescribed within 24 hours before hospital arrival or within 3 hours after hospital arrival" (Box 1, Appendix 3 [www.cmaj.ca/cgi/content/full /179/9/909/DC2]) is a modification of the indicator from the initial set of Canadian quality indicators — "ASA prescribed within 6 hours after hospital arrival" 6 - and differs from the US indicator of "ASA within 24 hours of hospital arrival." The panel felt that ASA should be administered as soon as possible after the patient's arrival at hospital but noted that there was no specific evidence to justify stipulation of administration within 3 hours after arrival — this time window was selected on the basis of expert opinion.

The 7 nonpharmacologic indicators (Box 1, Appendix 3 [www.cmaj.ca/cgi/content/full/179/9/909/DC2]) serve to update and replace several from the initial set of quality indicators. ${ }^{6}$ These 7 indicators focus on rapid identification and treatment of patients with ST-segment elevation myocardial infarction, risk stratification, assessment of left ventricular function, as well as
Box 1: In-hospital process-of-care, outcome and system indicators* for acute myocardial infarction

\section{Pharmacologic process-of-care indicators}

- ASA within 24 hours before hospital arrival or within 3 hours after hospital arrival

- ASA prescribed at hospital discharge

- $\beta$-Blocker prescribed at hospital discharge

- ACE inhibitor or angiotensin-receptor blocker prescribed at hospital discharge

- Statin prescribed at hospital discharge

- Fibrinolytic therapy within 30 minutes after hospital arrival

Nonpharmacologic process-of-care indicators

- Electrocardiogram (ECG) within 10 minutes after hospital arrival

- Primary percutaneous coronary intervention within 90 minutes after hospital arrival

- Reperfusion therapy in eligible patients with ST-segment elevation myocardial infarction

- Risk stratification (i.e., cardiac catheterization, exercise stress testing, perfusion imaging or stress echocardiography)

- Assessment of left ventricular function

- Smoking cessation advice, counselling or therapy during hospital stay

- Referral to cardiac rehabilitation

\section{Outcome indicator}

- In-hospital mortality

\section{System indicators}

- Fibrinolytic therapy within 60 minutes after call for emergency medical services

- Primary percutaneous coronary intervention within 120 minutes after call for emergency medical services

- Prehospital 12-lead ECG

Note: $\mathrm{ACE}=$ angiotensin-converting enzyme, ASA = acetylsalicylic acid

*These indicators are measurable from chart review. 
counselling about smoking cessation and referral to cardiac rehabilitation. ${ }^{15}$ A new indicator, "electrocardiogram (ECG) within 10 minutes after hospital arrival" (Box 1, Appendix 3 [www.cmaj.ca/cgi/content/full/179/9/909/DC2]) was deemed important because of its role in determining the type of myocardial infarction and linkage to subsequent steps in the care process, such as provision of fibrinolytic therapy or primary percutaneous coronary intervention. Arrival at hospital was defined as triage time or registration time, whichever came first.

Panelists recommended that the majority of patients with acute myocardial infarction undergo risk stratification with one of the following procedures: cardiac catheterization; exercise stress testing; perfusion imaging; or stress echocardiography (Box 1, Appendix 3 [www.cmaj.ca/cgi/content/full/179/9 /909/DC2]). The panelists did not specify a particular timeframe for cardiac catheterization, since benchmarks have already been developed for patients with acute coronary syndromes by the Canadian Cardiovascular Society as part of its Access to Care initiative, ${ }^{16}$ and there were concerns about logistical challenges in operationalizing a complex, time-

Box 2: Out-of-hospital process-of-care and outcome indicators* for acute myocardial infarction

Pharmacologic process-of-care indicators

- Prescription for $\beta$-blocker filled within 30 days after discharge

- Prescription for $\beta$-blocker filled within 90 days after discharge

- Adherence to $\beta$-blocker therapy 1 year after discharge

- Prescription for ACE inhibitor or angiotensin-receptor blocker filled within 30 days after discharge

- Prescription for ACE inhibitor or angiotensin-receptor blocker filled within 90 days after discharge

- Adherence to ACE inhibitor or angiotensin-receptor blocker therapy 1 year after discharge

- Prescription for statin filled within 30 days after discharge

- Prescription for statin filled within 90 days after discharge

- Adherence to statin therapy 1 year after discharge

Nonpharmacologic process-of-care indicators

- Physician visit within 4 weeks after discharge

- Median wait time (in days) for cardiac catheterization after myocardial infarction

- Median wait time (in days) for percutaneous coronary intervention after myocardial infarction

- Median wait time (in days) for coronary artery bypass graft surgery after myocardial infarction

\section{Outcome indicators}

- 30-day mortality

- 1-year mortality

- 30-day readmission rate because of acute myocardial infarction

- 1-year readmission rate because of acute myocardial infarction

- 30-day readmission rate because of congestive heart failure

- 1-year readmission rate because of congestive heart failure

- 30-day readmission rate because of unstable angina

- 1-year readmission rate because of unstable angina

Note: $\mathrm{ACE}=$ angiotensin-converting enzyme

*These indicators are measurable using administrative data. dependent indicator. The panel recognized that many patients would undergo semi-urgent percutaneous coronary intervention or coronary artery bypass graft surgery based on the findings of the cardiac catheterization; however, the panel felt that it would be overly complex to operationalize an indicator for these interventions after an acute myocardial infarction.

The panel also discussed the application of the indicator "primary percutaneous coronary intervention within 90 minutes after hospital arrival" to transferred patients. It strongly recommended that this indicator be measured for both transferred patients and nontransferred patients, albeit separately. With the benefits of primary percutaneous coronary intervention being increasingly recognized, more patients are being transferred to centres with such treatment capabilities. In the panel's view, the same goal for the treatment timeframe should apply to both transferred and nontransferred patients receiving primary percutaneous coronary intervention (i.e., door-to-balloon time of 90 minutes or less). However, it should be measured separately in these 2 groups of patients. Further, the panel noted that, although the door-to-balloon time has traditionally been reported as a median value, the indicator sets a target for all eligible patients to receive primary percutaneous coronary intervention within 90 minutes after arrival at hospital, not for a median door-to-balloon time of $90 \mathrm{~min}$ utes or less, as it has been interpreted in some settings. Hospitals that transfer patients from their emergency department to a facility for percutaneous coronary intervention may wish to measure the time from arrival at hospital to transfer, recognizing the importance of this phase in the door-to-reperfusion process.

\section{Process-of-care and outcome indicators - administrative data}

The panel unanimously recommended 13 out-of-hospital process-of-care indicators (9 pharmacologic and 4 nonpharmacologic) based on administrative data (Box 2). These indicators were similar to those in the original set of indicators. ${ }^{6}$

The 9 pharmacologic indicators focus on secondary prevention in the form of prescriptions filled for medications such as $\beta$-blockers, ACE inhibitors or angiotensinreceptor blockers, and statins at various points. The 4 nonpharmacologic indicators measure wait times for procedures and the proportion of patients who have a physician visit within 4 weeks after discharge.

There were also 8 outcome indicators that measure cardiac-related readmission rates and mortality rates at different points. More explicit definitions of these indicators are provided in the initial publication of quality indicators. ${ }^{6}$ Because in-hospital 
mortality is sensitive to a patient's length of stay, the panel recognized that 30-day mortality might be a preferable indicator. However, it is a difficult indicator for individual hospitals to track, because the patient is often no longer within the hospital setting. Furthermore, most deaths in the short term after an acute myocardial infarction occur during the index in-hospital stay.

\section{System indicators}

Three new system indicators were included (Box 1, Appendix 3 [www.cmaj.ca/cgi/content/full/179/9/909/DC2]). The first system indicator, "fibrinolytic therapy within 60 minutes after call for emergency medical services," is similar to the performance measure used in the United Kingdom under the National Service Framework for Coronary Heart Disease. ${ }^{8}$ The second system indicator, "primary percutaneous coronary intervention within 120 minutes after call for emergency medical services," identifies a call-to-intervention time of 120 minutes. These indicators focus on assessing the collaborative responsiveness of the emergency medical services and the hospital to the initial call for help. The third indicator — "prehospital 12-lead ECG" - assesses the proportion of patients who received a 12-lead ECG in the field and the capability of the emergency medical services to provide this service. Not all jurisdictions in Canada currently have ambulances with 12-lead ECG equipment, although this is expected to change in the years ahead.

These 3 system indicators incorporate important aspects of the period of prehospital care and represent an important opportunity to reduce call-to-intervention time for patients with ST-segment elevation myocardial infarction.

\section{Discarded indicators}

The panel discarded several indicators from the initial set. ${ }^{6}$ The in-hospital process-of-care indicator " $\beta$-blocker within 12 hours of hospital admission" was discarded because recent evidence suggested that it might be harmful in the acute phase of myocardial infarction in some patients. ${ }^{17}$

The indicator "clopidogrel administered either acutely or at hospital discharge" was considered as a potential new indicator but was ultimately discarded because of a lack of consensus as to whether there was sufficient evidence to warrant its recommendation for all patients with acute myocardial infarction.

Another process-of-care indicator from the initial set, "lipid sample obtained within 24 hours of admission," " was discarded because the panel concluded that most patients should be given statin therapy regardless of their lipid levels, with blood levels to be monitored during follow-up.

Although not defined as an indicator, the in-hospital use of anticoagulants was discussed. The panel concluded that anticoagulants (e.g., heparin, low-molecular-weight heparin) are an important component of care. However, it refrained from creating an indicator for anticoagulant use because of the complex range of agents, regimens and relative indications that would need to be addressed in the definition, implementation and measurement of such an indicator.

\section{Benchmarks}

Although benchmarks varied in the initial set of quality indicators (from $70 \%$ to $90 \%$ ), ${ }^{6}$ the panel unanimously selected $90 \%$ as the target benchmark in the updated set of process-of-care indicators for ideal patients. However, it noted that, for some hospitals and indicators, a $90 \%$ target may be unrealistic or difficult to achieve relative to current performance levels. In these instances, hospitals are encouraged to adopt annual improvement targets set at increments of $10 \%$. For example, if a hospital's performance on an indicator is currently $70 \%$, the target for the next year would be $80 \%$ for that indicator. At the same time, the panel felt it was important to select benchmarks that are high enough to motivate hospitals to improve and work toward achieving the highest possible quality of care.

The panel did not explicitly recommend target benchmarks for the outcome indicators. It recognized that outcomes are dependent not only on the quality of care provided but also on patient characteristics that may be out of a hospital's direct control. Nevertheless, tracking outcomes is important, and hospitals should strive for continuous improvement in all outcome measures over time.

\section{Discussion}

\section{Use and impact of indicators}

The initial set of indicators have been used in a variety of settings since their publication in $2003 .{ }^{6}$ The indicators served as the basis for report cards for quality of cardiac care that were developed for Ontario hospitals as part of the Enhanced Feedback for Effective Cardiac Treatment (EFFECT) Study. Many Ontario hospitals developed initiatives to improve their performance after the first round of report cards were publicly released from this study. ${ }^{18}$ The initial indicators have also been used in other initiatives such as the Saskatchewan Health Quality Council hospital-specific, province-wide report card for quality of care of acute myocardial infarction ${ }^{19}$ and by Canadian hospitals participating in the "Safer Health Care Now!" acute myocardial infarction quality-improvement initiative..$^{20}$ The application of the indicators in these types of initiatives has provided valuable insight into their feasibility, ease of use, availability of required data and their ability to identify variation in care across hospitals. Experience from these initiatives demonstrates the need for indicators that are easy to use, with definitions that are clear and concise. This will support consistent use and application of the indicators and help to minimize chart-abstraction time and data-collection costs.

The definitions of, and inclusion and exclusion criteria for, the updated set of indicators have been worded specifically to reflect changes in practice guidelines and scientific evidence since the first set of indicators were published. Although the updated indicators are most likely going to be adopted in the Canadian health care system, they should also be useful in other countries, particularly jurisdictions where quality-ofcare indicators are not widely used.

There is increasing evidence that hospitals with better performance on these types of quality-of-care indicators have lower mortality rates. ${ }^{21}$ Thus, improving performance on these indicators has the potential to save many lives. ${ }^{21}$

Different users of these indicators may wish to tailor them to their own settings and may not measure all of the indicators on a regular basis. 


\section{Implementation strategies and challenges}

For indicators to have maximal impact on the health care system, clinicians and hospitals need to commit adequate time and resources to measuring their performance on the indicators and developing strategies for achieving optimal performance. For busy clinicians and financially strapped hospitals, quality-improvement activities may not always be a high priority. In most Canadian provinces, quality improvement has been a voluntary activity left to individual clinicians and hospitals. However, this may change as the public and policymakers demand greater accountability for health care spending and corresponding outcomes.

In both the United States and the United Kingdom, hospitals are mandated by government, private payers and hospital accreditation bodies to collect and publicly report their performance on a number of quality-of-care indicators for acute myocardial infarction as a condition of reimbursement. In an increasing number of cases, there are "pay for performance" strategies in place whereby hospitals that achieve the best performance on indicators receive financial rewards. ${ }^{22}$ The adoption of similar policies in Canada would rapidly increase the interest in, and uptake of, these indicators and likely improve patient outcomes.

To assist Canadian hospitals with the implementation of these quality-of-care indicators, the Canadian Cardiovascular Outcomes Research Team investigators will work in collaboration with national and local initiatives (e.g., the "Safer Healthcare Now!" campaign) to develop measurement tools such as data-collection sheets and web-based clinical registries that will enable hospitals to measure their performance on these indicators. ${ }^{20}$ The "Safer Healthcare Now!" campaign currently has more than 120 hospital teams from across Canada enrolled in its initiative to improve the delivery of care for acute myocardial infarction using evidence-based indicators (Virginia Flintoft, Department of Health Policy, Management and Evaluation, University of Toronto, Toronto, Ontario: personal communication, 2008). Data from the EFFECT study have shown that hospitals that use standardized tools (e.g., admitting orders) have higher rates of performance on quality indicators. Therefore, we recommend that hospitals consider developing or modifying standardized admission orders, care pathways and discharge plans to incorporate the aspects of care covered by these indicators. ${ }^{18}$

This updated set of indicators will also be presented at various scientific meetings. A slide show will be developed to help those who wish to do presentations on these indicators at their local hospitals. As resources for using these indicators are developed, they will be made available by the Canadian Cardiovascular Outcomes Research Team on its website (www.ccort.ca).

\section{Limitations}

We recognize that these quality-of-care indicators have certain limitations. Failure to provide an identified element of care to a given patient may not necessarily represent suboptimal care - the need for clinical judgment in the care of each patient remains. There may also be extenuating circumstances that might influence a clinician's judgment. These circum- stances may not be captured or documented in the patient chart, which is why the panel did not recommend a target benchmark of $100 \%$ for individual indicators. The updated set of indicators have focused predominantly on underuse of interventions. However, the overuse of potentially harmful interventions (e.g., calcium-channel blockers in patients with left ventricular dysfunction) is also an important quality-ofcare issue. Finally, quality of care is a multidimensional construct, and certain important aspects of care such as the quality of patient education and counselling are not captured by these indicators, which focus on aspects of care that can be reliably and easily measured.

\section{Conclusion}

The initial set of indicators of quality of care for patients with acute myocardial infarction that was published in $2003^{6}$ has been updated to reflect current evidence and practice guidelines, as interpreted by an expert multidisciplinary panel of members from across Canada. These indicators are designed to be tools to measure the quality of care objectively and to identify areas where processes of care and patient outcomes can be improved. The first set of indicators has had a positive impact on the Canadian health care system. One hopes that this updated set will have a similarly positive effect.

Performance measures are not intended to be an end unto themselves, but rather a critical first step in measuring and improving the care of patients. We suggest that clinicians and hospitals, working collaboratively, use the indicators to measure their performance, assess the results and implement strategies to ensure that every patient with acute myocardial infarction gets the best possible care and outcomes.

This article has been peer reviewed.

Competing interests: Michel Love, a member of the Canadian Cardiovascular Outcomes Research Team / Canadian Cardiovascular Society Acute Myocardial Infarction Quality Indicator Panel, has received speaker fees from SanofiAventis, Bristol-Myers Squibb, GlaxoSmithKline and Pfizer, and travel assistance from Schering-Plough to attend the 2007 American Heart Association meeting. No competing interests declared by the authors or other panel members.

Contributors: Jack Tu conceived the idea for this project and obtained the funding. Laila Khalid led the review of the background literature. Jack Tu, Laila Khalid, Linda Donovan and Dennis Ko drafted the manuscript. Each of the members of the expert panel revised the manuscript critically for important intellectual content and approved the final version for publication. All of the authors participated significantly in the design, development and refinement of the quality indicators and approved the final version of the manuscript for publication.

Funding: Supported by a Canadian Institutes of Health Research (CIHR) Team Grant in cardiovascular outcomes research and a grant from the Heart and Stroke Foundation of Ontario. Dr. Tu is supported by a Canada Research Chair in Health Services Research and a Career Investigator Award from the Heart and Stroke Foundation of Ontario. Dr. Ko is supported by a ClinicianScientist Award from the Heart and Stroke Foundation of Ontario.

\section{REFERENCES}

1. Fox KA, Goodman SG, Klein W, et al. Management of acute coronary syndromes. Variations in practice and outcome: findings from the Global Registry of Acute Coronary Events (GRACE). Eur Heart J 2002;23:1177-89.

2. Fox KA, Steg PG, Eagle KA, et al. Decline in rates of death and heart failure in acute coronary syndromes, 1999-2006. JAMA 2007;297:1892-900. 
3. Eagle KA, Nallamothu BK, Mehta RH, et al. Trends in acute reperfusion therapy for ST-segment elevation myocardial infarction from 1999 to 2006: We are getting better but we have got a long way to go. Eur Heart J 2008;29:609-17.

4. Lenfant $C$. Shattuck lecture. Clinical research to clinical practice: Lost in translation? N Engl J Med 2003;349:868-74.

5. Spertus JA, Eagle KA, Krumholz HM, et al. American College of Cardiology and American Heart Association methodology for the selection and creation of performance measures for quantifying the quality of cardiovascular care. Circulation 2005;111:1703-12.

6. Tran CT, Lee DS, Flintoft VF, et al. CCORT/CCS quality indicators for acute myocardial infarction care. Can J Cardiol 2003;19:38-45.

7. Krumholz HM, Anderson JL, Brooks NH, et al. ACC/AHA clinical performance measures for adults with ST-elevation and non-ST-elevation myocardial infarction: a report of the American College of Cardiology/American Heart Association Task Force on Performance Measures (Writing Committee to Develop Performance Measures on ST-Elevation and Non-ST-Elevation Myocardial Infarction). Circulation 2006;113:732-61.

8. Department of Health. Coronary heart disease: national service framework for coronary heart disease - modern standards and service models. London (UK): The Department; 2000. Available: www.dh.gov.uk/en/Publicationsandstatistics /Publications/PublicationsPolicyAndGuidance/DH_4094275 (accessed 2008 Aug 22).

9. Idanpaan-Heikkila UM, Lambie L, Mattke S, et al. Selecting indicators for the quality of cardiac care at the health system level in Organization for Economic Cooperation and Development countries. Int J Qual Health Care 2006;18(Suppl 1):39-44.

10. Normand SL, McNeill BJ, Peterson LE, et al. Eliciting expert opinion using the Delphi technique: identifying performance indicators for cardiovascular disease. Int J Qual Health Care 1998;10:247-60.

11. Antman EM, Anbe DT, Armstrong PW, et al. ACC/AHA guidelines for the management of patients with ST-elevation myocardial infarction; A report of the American College of Cardiology/American Heart Association Task Force on Practice Guidelines (Committee to Revise the 1999 Guidelines for the Management of patients with acute myocardial infarction). J Am Coll Cardiol 2004;44:E1-211.

12. Anderson JL, Adams CD, Antman EM, et al. ACC/AHA 2007 guidelines for the management of patients with unstable angina/non-ST-Elevation myocardial infarction: a report of the American College of Cardiology/American Heart Association Task Force on Practice Guidelines (Writing Committee to Revise the 2002 Guidelines for the Management of Patients With Unstable Angina/Non-ST-Elevation Myocardial Infarction). J Am Coll Cardiol 2007;50:e1-157.

13. Antman EM, Hand M, Armstrong PW, et al. 2007 focused update of the ACC/AHA 2004 guidelines for the management of patients with ST-elevation myocardial infarction: a report of the American College of Cardiology/American Heart Association Task Force on Practice Guidelines. J Am Coll Cardiol 2008;51:210-47.

14. Spertus JA, Radford MJ, Every NR, et al. Challenges and opportunities in quantifying the quality of care for acute myocardial infarction: summary from the Acute Myocardial Infarction Working Group of the American Heart Association/American College of Cardiology First Scientific Forum on Quality of Care and Outcomes Research in Cardiovascular Disease and Stroke. Circulation 2003;107:1681-91.

15. Thomas RJ, King M, Lui K, et al. AACVPR/ACC/AHA 2007 performance measures on cardiac rehabilitation for referral to and delivery of cardiac rehabilitation/ secondary prevention services. Circulation 2007;116:1611-42.

16. Graham MM, Knudtson ML, O'Neill BJ, et al. Treating the right patient at the right time: access to cardiac catheterization, percutaneous coronary intervention and cardiac surgery. Can J Cardiol 2006;22:679-83.

17. Chen ZM, Pan HC, Chen YP, et al. Early intravenous then oral metoprolol in 45,852 patients with acute myocardial infarction: randomised placebo-controlled trial. Lancet 2005;366:1622-32.
18. Tu JV, Donovan LR, Austin PC, et al. Quality of cardiac care in Ontario - phase I. Toronto (ON): Institute for Clinical Evaluative Sciences; 2004. Available: www.ices.on.ca/file/CCORT_EFFECT_Phase1_Report2_final.pdf (accessed 2008 Aug 22).

19. Chan BTB, Brossart BD, Huderma NRL, et al. Improving the quality of heart attack care in Saskatchewan: outcomes and secondary prevention. Saskatoon (SK): Health Quality Council; 2004.

20. Safer Healthcare Now. AMI Getting Started Kit: The Campaign; 2005. Available: www. saferhealthcarenow.ca/Default.aspx ?folderId=82\&contentId=266. (accessed 2008 Aug 22)

21. Peterson ED, Roe MT, Mulgund J, et al. Association between hospital process performance and outcomes among patients with acute coronary syndromes. JAMA 2006;295:1912-20.

22. Lindenauer PK, Remus D, Roman S, et al. Public reporting and pay for performance in hospital quality improvement. N Engl J Med 2007;356:486-96.

Correspondence to: Dr. Jack V. Tu, Rm. G-106, Institute for Clinical Evaluative Sciences, 2075 Bayview Ave., Toronto ON M4N 3M5; fax 416 480-6048; tu@ices.on.ca

Members of the Canadian Cardiovascular Outcomes Research Team / Canadian Cardiovascular Society Acute Myocardial Infarction Quality Indicator Panel: Jack V. Tu MD PhD and Dennis T. Ko MD MSc (cochairs), Institute for Clinical Evaluative Sciences, Toronto, Ont.; Christopher E. Buller MD, Department of Medicine, Vancouver General Hospital, University of British Columbia, Vancouver, BC; Virginia F. Flintoft BN MSc, Department of Health Policy, Management and Evaluation, Faculty of Medicine, University of Toronto, Toronto, Ont.; Thao Huynh MD MSc, Research Institute of the McGill University Health Centre, Montréal, Que.; Cynthia A. Jackevicius PharmD MSc, Department of Health Policy, Management and Evaluation, Faculty of Medicine, University of Toronto, Toronto, Ont.; Laurie J. Lambert PhD, l'Agence d'évaluation des technologies et des modes d'intervention en santé, Montréal, Que.; Michael P. Love MB ChB MD, Department of Medicine, Queen Elizabeth II Health Sciences Centre, Dalhousie University, Halifax, NS; Michael J. Schull MD MSc, Department of Medicine, Sunnybrook Health Sciences Centre, Toronto, Ont.; Heather Sherrard BScN MHA, University of Ottawa Heart Institute, Ottawa, Ont.; Edward Y.K. Tsoi MB BCh, Department of Family Medicine, St. Joseph's Hospital of Estevan, Estevan, Sask.; and Alain Vadeboncoeur MD, Montréal Heart Institute, Montréal University, Montréal, Que. 